\title{
Rastreio cognitivo em estruturas residenciais para pessoas idosas no Concelho de Miranda do Corvo, Portugal
}

\author{
Cognitive screening for elderly people in long-term care \\ institutions in the Miranda do Corvo municipality, Portugal
}

Fernanda Daniel (https://orcid.org/0000-0002-2202-1123) 1,2

Vanessa Fernandes (https://orcid.org/0000-0003-0761-7313) ${ }^{2}$

Alexandre Silva (https://orcid.org/0000-0001-5163-9670) 1,3

Helena Espírito-Santo (http://orcid.org/0000-0003-2625-3754) ${ }^{2}$

${ }^{1}$ Centro de Estudos

e Investigação em

Saúde, Universidade de

Coimbra. Avenida Dias

da Silva 163. 3004-512

Coimbra Portugal.

fernanda.b.daniel@

gmail.com

${ }^{2}$ Centro Interdisciplinar de

Investigação Psicossocial,

Instituto Superior Miguel

Torga. Coimbra Portugal.

${ }^{3}$ Instituto de Contabilidade

e Administração de

Coimbra. Coimbra

Portugal.

\begin{abstract}
This study aimed to screen the cognitive profile elderly people living in long-term care institutions in the municipality of Miranda do Corvo by evaluating 174 participants with the Mini Mental State Examination (MMSE) $(n=96)$ and the clinical dementia diagnosis $(n=78)$. According to the MMSE, $41.7 \%$ of respondents had scores suggestive of cognitive impairment. The percentage rose to $67.8 \%(n=118)$ by adding the diagnosis of dementia reported in individual medical records to this result. The comparison of our results with those obtained nationwide showed that this proportion was significantly higher $(p<0.001)$. The educational level was a predictive factor for MMSE scores $(p=0.001)$. We can conclude that the high prevalence of suspected cognitive impairment and dementia revealed in our study should lead us to reflect on the quality of care provided and on the lack/scarcity of cognitive stimulation programs in long-term care institutions for seniors. Thus, it is imperative to implement regular cognitive assessment and to apply intervention programs for the preservation and improvement of the cognitive functioning of institutionalized elderly of deprived areas.

Key words Elderly, Institutionalization, Cognitive deficit, Cognitive screening.
\end{abstract}

Resumo Com o objetivo de efetuar o rastreio do perfil cognitivo dos residentes em Estruturas Para Idosos no Concelho de Miranda do Corvo, avaliaram-se 174 participantes recorrendo ao MiniMental State Examination (MMSE) ( $n=96)$ e ao diagnóstico de demência reportado nos prontuários dos pacientes $(n=78)$. Verificou-se, através do MMSE, que 41,7\% dos inquiridos apresentavam pontuações sugestivas de déficit cognitivo. Adicionando a este resultado o diagnóstico de demência reportado nos prontuários dos pacientes, a percentagem subiu para $67,8 \%(n=118)$. A comparação dos nossos resultados com os obtidos a nível nacional revelou que essa percentagem foi significativamente superior $(p<0,001)$. A escolaridade foi um fator preditivo da pontuação do MMSE ( $p=0,001)$. Conclui-se que a elevada prevalência de suspeita de déficit cognitivo e de demência revelada no nosso estudo deve remeter para a reflexão sobre a adequação dos cuidados prestados e sobre a ausência/escassez de programas de estimulação cognitiva nas estruturas residenciais para idosos. Nesse sentido, torna-se imperativo implementar avaliação cognitiva regular e instituir programas de intervenção que promovam a conservação $e$ melhoria do funcionamento cognitivo em pessoas idosas institucionalizadas de zonas desfavorecidas. Palavras-chave Pessoas idosas, Institucionalização, Déficit cognitivo, Rastreio cognitivo. 


\section{Introdução}

A discussão crítica acerca do envelhecimento tem sido fecunda, observando a investigação produzida desde o fim do século XX. O interesse crescente nesta temática foi previsto por Philippe Ariès em 1983, ao afirmar que quando o bulldozer acadêmico se pusesse em marcha, surgiria uma autêntica biblioteca sobre o envelhecimento ${ }^{1}$. Este interesse decorre do aumento da expressão numérica das pessoas idosas em consequência tanto da diminuição da natalidade como do aumento da esperança média de vida. Note-se que, em Portugal, a população idosa em 1960 era de 7,97\%, enquanto o último momento censitário de 2011 apresentava uma percentagem de $19,03 \%{ }^{2}$. Mais recentemente, as estimativas anuais da população residente evidenciam que a percentagem da população idosa continua a aumentar, situando-se em 2016 em 20,90\% ${ }^{3}$.

A reflexão sobre o envelhecimento explora um processo que não é unitário, mas complexo e vivido através de mudanças biológicas, psicológicas e sociais, tendendo a um aumento de complexidade com o avanço da idade. Como processo multidimensional e multideterminado, o envelhecimento está associado ao declínio natural de funções fisiológicas que influenciam o indivíduo ${ }^{4}$, de onde se destaca, a fragilidade ${ }^{5}$ e várias doenças físicas ${ }^{6}$. Está também relacionado com a necessidade de lidar com as frequentes perdas (e.g., reforma, diminuição de rede social pessoal), com a diminuição da plasticidade comportamental ${ }^{7}$, com a diminuição das interações sociais, com o aumento da seletividade dos relacionamentos sociais e, ainda, com alterações no comportamento emocional ${ }^{8,9}$.

O envelhecimento, enquanto processo progressivo de degradação, comum a todos os seres vivos e que não está obrigatoriamente associada à existência de doença, ocorre de forma diferente de indivíduo para indivíduo. Por esse fato, a investigação gerontológica começou, no final do século XX, a divulgar o conceito de envelhecimento bem-sucedido, consistindo na combinação de uma baixa probabilidade de doença, na manutenção das funções físicas e cognitivas e no empenhamento em atividades produtivas, a par da manutenção de relações interpessoais ${ }^{10}$. Evidencia-se a multidimensionalidade do conceito de envelhecimento bem-sucedido, relacionando -o com a adaptação ao funcionamento fisiológico (físico, cognitivo e mental).

O envolvimento social, o sistema de suporte, a presença de recursos pessoais (resiliência, coping e independência/autonomia), fatores extrínsecos (ambiente e finanças) e o bem-estar (estado afetivo e satisfação com a vida) são dimensões que estão também reportadas em maior ou menor grau num envelhecimento bem-sucedido ${ }^{11}$. Se analisarmos coortes de idades, verificamos diferenças no desempenho, avaliado através de testes neuropsicológicos, na capacidade funcional e nos diferentes domínios cognitivos. A avaliação neuropsicológica denuncia um declínio da memória de trabalho com o aumento da idade, enquanto no caso do envelhecimento patológico são reportadas, além de alterações na memória, alterações na linguagem e funcionamento executivo ${ }^{12-14}$.

No que respeita ao déficit cognitivo ligeiro (precursor potencial de demência) e à síndrome demencial, a idade é comumente considerada um dos principais fatores de risco. Em Portugal, estima-se que existam 182.526 pessoas com demência, representando $1,71 \%$ do total da população ${ }^{15}$.

Segundo Buntinx et al. ${ }^{16}$, a demência é uma desordem progressiva, insidiosa, apresentando um quadro clínico inicial muito diverso, podendo, inclusive, mimetizar um quadro depressivo. No entanto, no que concerne ao potencial preditivo da depressão na demência, os autores não demonstram consenso ${ }^{17,18}$. Com a evolução da demência, surge a progressiva perda da capacidade funcional, instalando-se a dependência.

Em face destas perdas, a necessidade de ajuda e apoio de terceiros torna-se crucial. Quando a rede familiar se encontra impossibilitada de responder a estas necessidades, em virtude de vários condicionantes, surge a necessidade de recorrer a respostas sociais dirigidas a esta população. A integração nestas respostas sociais, mais concretamente numa Estrutura Residencial para Idosos, é um processo que não é, nem simples nem fácil, na medida em que implica a adaptação a um novo cenário com novos rituais organizativos e novos papéis ${ }^{19,20}$. Por este fato, a par da dificuldade em manter a integridade da rede social, diferentes estudos reportam que as pessoas idosas preferem majoritariamente permanecer nas suas $\operatorname{casas}^{21-26}$.

Apesar de o déficit cognitivo ser um fator que prediz a institucionalização, muitas das instituições sociais não se encontram estruturadas para esta realidade quer ao nível organizacional, quer ao nível dos recursos humanos ${ }^{23}$, pois apresentam um modelo organizativo que favorece a sobreproteção, não estimulando o desempenho físico e cognitivo ${ }^{20}$. A própria legislação que define as condições a que devem obedecer estas estruturas residenciais (Portaria no 67/2012 de 
21 de março) define uma equipe técnica restrita. Nessa legislação, para além do diretor técnico, só é exigido a existência de um(a) animador(a) sociocultural, educador(a) social ou técnico(a) de geriatria a tempo parcial por cada 40 residentes e um(a) enfermeiro(a) por cada 40 residentes (itálico nosso).

A investigação sedimentada nesta área refere aos efeitos negativos da sobreproteção e da ausência de controle por parte dos residentes, o que reforça comportamentos dependentes, sem que isto signifique a falta de competência real do residente $^{27}$. A ausência de controle ${ }^{28}$ e de estimulação cognitiva poderão funcionar como aceleradores do declínio cognitivo ${ }^{29}$, a par das características desta coorte geracional. Parece-nos por isso essencial executar, nas estruturas residenciais para idosos, uma avaliação objetiva das funções cognitivas e proceder a essa avaliação o mais precocemente possível. A par da avaliação cognitiva, uma avaliação multidimensional deve ser ativada para que a suspeita de demência seja progressivamente confirmada ${ }^{30-32}$ e as suas consequências (e.g., alterações na funcionalidade) sejam devidamente acompanhadas ${ }^{33}$. Importa, contudo, referir que existem vários instrumentos de avaliação do estado mental, mas não existem instrumentos de padrão de ouro para o diagnóstico ${ }^{34}$. É relevante mencionar que variáveis como a idade e a escolaridade interferem com o desempenho em testes neuropsicológicos ${ }^{35}$.

A presente investigação teve como objetivos: (1) identificar o perfil cognitivo das pessoas que se encontravam em Estruturas Residenciais para Idosos no concelho de Miranda do Corvo, utilizando como ferramentas as dimensões do MiniMental State Examination (MMSE); (2) estudar o impacto da idade e escolaridade sobre o estado cognitivo; (3) determinar as prevalências do déficit cognitivo (MMSE) e do diagnóstico de demência (prontuários dos pacientes) e (4) comparar os valores de prevalência com uma amostra representativa da população portuguesa.

\section{Métodos}

O estudo, transversal, incluiu a resposta social "Estruturas Residenciais para Idosos" (ERPIs) do Concelho de Miranda do Corvo, tendo sido aprovada pelo Departamento de Investigação \& Desenvolvimento do Instituto Superior Miguel Torga. A informação sobre as ERPIs do Concelho foi efetuada recorrendo à base de dados da Carta Social Portuguesa ${ }^{36}$. Este concelho, selecionado pela proximidade geográfica aos investigadores, ocupa uma área de aproximadamente $126,4 \mathrm{~km}^{2}$, distribuída por quatro freguesias consideradas, segundo a Portaria no 22/2015 de 5 de fevereiro, zonas desfavorecidas de montanha. O contato com as ERPIs iniciou-se enviando um ofício em correio postal com a descrição detalhada do estudo. As ERPIs que aceitaram integrar este estudo, obedeciam ao estipulado pela legislação ao nível das equipas técnicas, das condições de funcionamento e de instalação.

O único critério de seleção foi a idade - igual ou superior a 65 anos. Nestas condições, encontravam-se 174 pessoas, tendo sido inquiridas somente 96 pessoas porque as restantes 78 pessoas, além de terem registrado diagnóstico de demência no prontuário do paciente, não apresentavam condições para serem avaliadas, nomeadamente sofriam de perda de linguagem falada, dificuldades na compreensão da linguagem e/ou de dificuldades motoras. Assim, a informação sociodemográfica destas 78 pessoas foi também recolhida nos respetivos prontuários dos pacientes.

Após assinatura da declaração de consentimento informado pelo próprio ou pelos cuidadores/familiares, uma equipe de psicólogos treinados no âmbito do projeto Trajetórias do envelhecimento, administraram durante três meses, no Concelho de Miranda do Corvo, um breve questionário sociodemográfico e clínico e o $\mathrm{Mi}$ ni-Mental State Examination/Avaliação Breve do Estado Mental (MMSE).

Os prontuários do paciente nas ERPIs, onde foi efetuada a coleta de dados, incluem informação sociodemográfica, familiar, situação social e de saúde. A informação extraída foi o sexo, idade, estado civil, escolaridade e o diagnóstico de demência.

O questionário sociodemográfico e clínico incluiu as questões sobre o sexo (codificado em feminino e masculino), idade (categorizada em duas faixas etárias: [65-75 [e $\geq 75$ anos]); estado civil (codificado em solteiro/a, casado/a, separado/a ou divorciado/a e viúvo/a), escolaridade [sem escolaridade, escolaridade inferior à $4^{\mathrm{a}}$ classe; $4^{\mathrm{a}}$ classe ( $1^{\mathrm{o}}$ ano do ensino básico); $5^{\circ}$ ao $6^{\circ}$ ano ( $2^{\circ}$ ciclo do ensino básico); $7^{\circ}$ ao $9^{\circ}$ ano ( $3^{\circ}$ ciclo do ensino básico); 12º ano (ensino secundário); curso médio e curso superior].

O MMSE é um instrumento que permite avaliar as funções cognitivas e rastrear o déficit cognitivo. Desenvolvido em 1975 por Folstein e colaboradores ${ }^{37}$, é um dos instrumentos mais utilizados e estudados ${ }^{38,39}$. É um teste simples e de rápida aplicação (5-15 minutos), permitindo 
avaliar a orientação (temporal e espacial); memória a curto prazo (retenção e evocação); atenção e cálculo (subtração em série); linguagem (nomear objetos, repetir frase, cumprir três comandos, ler-cumprir um comando e escrever uma frase); e a capacidade visuoconstrutiva (copiar dois pentágonos intersetados). A sua classificação final pode variar entre 0 e 30 pontos. Embora este instrumento possua uma série de recursos úteis para a prática clínica, não suporta, segundo Guerrei$\mathrm{ro}^{40}$, a responsabilidade de um diagnóstico formal. Ainda assim, através do MMSE é possível determinar a presença de déficit cognitivo através dos seus pontos de corte.

Os pontos de corte deste instrumento são diferenciados consoante o nível de escolarização dos sujeitos ${ }^{41}$. Neste sentido, considera-se existir déficit cognitivo, quando a pontuação for igual ou inferior a 15 pontos, no caso de analfabetos; 22 pontos em indivíduos com onze anos ou menos anos de escolaridade; e 27 pontos para aqueles com uma escolaridade superior a onze anos ${ }^{41}$. Não obstante, importa salientar que o MMSE possui limitações, incluindo a possibilidade de a idade, escolaridade e saúde poderem influenciar o desempenho cognitivo no teste ${ }^{42}$. Portanto, podemos afirmar que a sua precisão vai depender de possíveis ajustamentos dos pontos de corte, principalmente para o nível educacional, porque a utilização de um ponto de corte pode levar a falsos positivos entre os pacientes com baixos níveis de escolaridade, bem como resultados falsos negativos entre aqueles que possuem elevado nível educacional ${ }^{37}$.

Assim, a nossa variável de desfecho - estado cognitivo - foi definida com base nos pontos de corte definidos para a população portuguesa do MMSE e com base na informação médica recolhida nos prontuários dos pacientes.

Preliminarmente, determinou-se o coeficiente alfa de Cronbach para o estudo da fidelidade do MMSE. No que respeita à descrição univariada das variáveis, recorremos à análise das frequências, a medidas de tendência central e dispersão. $\mathrm{O}$ teste de ajustamento do qui-quadrado foi utilizado para verificar se a distribuição das frequências observadas dos dados se ajustava a um modelo teórico predeterminado, no caso presente à população de Portugal e do Estudo do Perfil do Envelhecimento da População Portuguesa. Recorremos ao teste de independência do qui-quadrado com correção de continuidade de Yates para explorar a relação entre duas variáveis categóricas. Para avaliar se a idade e a escolaridade prediziam as pontuações determinadas pelo
MMSE, recorremos a uma análise de regressão linear. Definiu-se para o estudo um nível de significância de 5\%.

\section{Resultados}

Os dados relativos à caracterização sociodemográfica foram recolhidos através da consulta dos prontuários dos pacientes e do questionário criado para o efeito.

Assim, verificamos que a maioria era mulher $(79,3 \%)$, tendo o teste de ajustamento do qui-quadrado indicado que a sua proporção foi significativamente diferente da encontrada na população de Miranda do Corvo de idade igual ou superior a 65 anos $^{43}$ (mulheres: $n_{\text {amostral }}=138$ e $N_{\text {populacional }}=1672$ vs. homens: $n_{\text {amostral }}=36$ e $N_{p o-}$ $\left.\underset{\text { pulacional }}{\text { populacional }}=1148 ; \chi^{2}=28,89, g l=1, p<0,001\right)$. No que respeita à idade, verificou-se uma média de $84,22(D P=7,58)$, tendo a maioria das pessoas idades iguais ou superiores aos 75 anos $(86,80 \%)$. De igual forma, a proporção etária foi significativamente distinta da população idosa de Miranda do Corvo ${ }^{43}$ (65-74 anos: $n_{\text {amostral }}=20$ e $N_{\text {populacional }}=$ $1424 ; \geq 75$ anos: $n_{\text {amostral }}=151$ e $N_{\text {populacional }}=1396$; $>65$ anos, mas sem especificação da idade: $n=3$; $\left.\chi^{2}=102,98, g l=1, p<0,001\right)$.

Acerca do estado civil, a maioria das pessoas era "viúva" (51,7\%), sendo essa percentagem superior quando comparada com a população idosa de Miranda do Corvo ${ }^{43}$ (solteiro/a: $n_{\text {amostral }}$ $=16$ e $N_{\text {populacional }}=149$; casado/a: $n_{\text {amostral }}=47 \mathrm{e}$ $N_{\text {populacional }}=1698$; divorciado/a: $n_{\text {amostral }}=8$ e $N_{\text {po. }}$ $\begin{gathered}\text { populacional } \\ \text { pulacional }\end{gathered}=89$; viúvo/a: $n_{\text {amostral }}=90 \mathrm{e} N_{\text {populacional }}=$ 884; Sem informação: $n_{\text {amostral }}=13 ; \chi^{2}=64,97, g l$ $=3, p<0,001)$. Finalmente, na escolaridade predominou a categoria "analfabeto" $(44,8 \%)$. Para poder comparar as categorias com as apresentadas no Instituto Nacional de Estatística relativas à população idosa de Miranda do Corvo ${ }^{43}$, tivemos de fundir categorias. Assim, a proporção nas distintas categorias foi significativamente diferente (sem escolaridade [inclui analfabeto e escolarida-

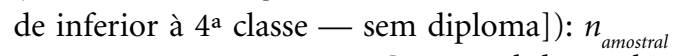
$=110$ e $N_{\text {populacional }}=1048$; Com nível de escolaridade completo: $n_{\text {amostral }}=51 \mathrm{e} N_{\text {populacional }}=1772$; Sem informação: $n_{\text {amostral }}=13 ; \chi^{2}=66,94, g l=1$; $p<0,001)$.

No estudo da fidelidade do MMSE, a consistência interna através do coeficiente alfa de Cronbach $(\alpha=0,73)$ indicou uma fidelidade aceitável segundo Peterson ${ }^{44}$ para instrumentos administrados no modo de hetero-administração.

Quanto ao primeiro objetivo, observamos 
na Tabela 1 as pontuações obtidas nas seis aptidões avaliadas pelo MMSE. As aptidões com maior número de acertos foram a Retenção e a Linguagem (94\% e 80\%, respectivamente). Na Linguagem, o item "Nomear o objeto Relógio" foi acertado por todos os respondentes. A aptidão com menor número de acertos foi a capacidade visuoconstrutiva (21\%) e a evocação da palavra "bola" com 34\% de acertos.

Para determinar se a idade e a escolaridade tiveram impacto na pontuação no MMSE efetuamos uma regressão linear. Como se pode obser- var na Tabela 2, o único preditor das pontuações no MMSE foi a variável escolaridade que apresentou uma contribuição estatisticamente significativa para o modelo $(p=0,001)$.

Ao dicotomizarmos as pontuações no MMSE nas categorias "presença" ou "ausência de déficit" e ao juntar os dados obtidos com o diagnóstico de demência extraído dos prontuários do paciente, observa-se que a proporção de pessoas com pontuações sugestivas de déficit cognitivo foi superior em todos os níveis de habilitações (Tabela 3), não havendo, por conseguinte, associação en-

Tabela 1. Pontuações Médias e Percentagem dos Acertos nas Respostas aos Itens do Mini-Mental State Examination (MMSE) $(\mathrm{n}=96)$

\begin{tabular}{|c|c|c|c|}
\hline \multicolumn{2}{|l|}{ MMSE } & $\%$ de acertos & $\mathbf{M}(\mathbf{D P})^{*}$ \\
\hline \multicolumn{2}{|c|}{ Orientação (máximo - 10 pontos) } & 14,6 & $0,72(0,23)$ \\
\hline \multicolumn{2}{|c|}{ Em que ano estamos } & 39,6 & $0,40(0,49)$ \\
\hline \multicolumn{2}{|l|}{ Em que mês estamos } & 75,0 & $0,75(0,44)$ \\
\hline \multicolumn{2}{|l|}{ Em que dia do mês estamos } & 31,3 & $0,31(0,47)$ \\
\hline \multicolumn{2}{|c|}{ Em que dia da semana estamos } & 81,3 & $0,81(0,39)$ \\
\hline \multicolumn{2}{|c|}{ Em que estação do ano estamos } & 77,1 & $0,77(0,42)$ \\
\hline \multicolumn{2}{|c|}{ Em que país estamos } & 93,8 & $0,94(0,24)$ \\
\hline \multicolumn{2}{|l|}{ Em que distrito vive } & 81,3 & $0,81(0,39)$ \\
\hline \multicolumn{2}{|l|}{ Em que terra vive } & 91,7 & $0,92(0,28)$ \\
\hline \multicolumn{2}{|l|}{ Em que casa estamos } & 86,5 & $0,86(0,34)$ \\
\hline \multicolumn{2}{|l|}{ Em que andar estamos } & 61,5 & $0,61(0,49)$ \\
\hline \multicolumn{2}{|c|}{ Retenção - Repetição das palavras (máximo - 3 pontos) } & 84,4 & $0,94(0,15)$ \\
\hline \multicolumn{2}{|c|}{ Pera } & 94,8 & $0,95(0,22)$ \\
\hline \multicolumn{2}{|l|}{ Gato } & 93,8 & $0,94(0,24)$ \\
\hline \multicolumn{2}{|l|}{ Bola } & 93,8 & $0,94(0,24)$ \\
\hline \multicolumn{2}{|c|}{ Atenção e cálculo - Subtração em sequência (máximo - 5 pontos) } & 30,2 & $0,46(0,43)$ \\
\hline \multicolumn{2}{|c|}{ Evocação - Evocação das palavras (máximo - 3 pontos) } & 21,9 & $0,48(0,37)$ \\
\hline \multicolumn{2}{|c|}{ Pera } & 56,3 & $0,56(0,50)$ \\
\hline \multicolumn{2}{|l|}{ Gato } & 52,1 & $0,52(0,50)$ \\
\hline \multicolumn{2}{|l|}{ Bola } & 34,4 & $0,34(0,48)$ \\
\hline \multicolumn{2}{|c|}{ Linguagem (máximo - 8 pontos) } & 14,6 & $0,80(0,14)$ \\
\hline \multirow[t]{2}{*}{ Nomear objetos } & Relógio & 100,0 & $1,00(0,00)$ \\
\hline & Lápis & 96,9 & $0,97(0,18)$ \\
\hline \multicolumn{2}{|c|}{ Repetir frase 'o rato roeu a rolha' } & 68,8 & $0,69(0,47)$ \\
\hline \multirow[t]{3}{*}{ Obedecer a três comandos } & Pegar com mão direita & 80,2 & $0,80(0,40)$ \\
\hline & Dobrar ao meio & 92,7 & $0,93(0,26)$ \\
\hline & Colocar onde deve & 80,2 & $0,80(0,40)$ \\
\hline \multicolumn{2}{|c|}{ Ler e cumprir uma instrução 'feche os olhos' } & 81,3 & $0 ; 81(0,39)$ \\
\hline \multicolumn{2}{|c|}{ Escrever uma frase com sentido, com sujeito e verbo } & 38,5 & $0,39(0,49)$ \\
\hline \multicolumn{2}{|c|}{$\begin{array}{l}\text { Habilidade Construtiva (máximo - } 1 \text { ponto) } \\
\text { Desenhar dois pentágonos parcialmente sobrepostos }\end{array}$} & 20,8 & $0,21(0,41)$ \\
\hline
\end{tabular}


tre as habilitações e a presença/ausência de déficit cognitivo $\left[\chi^{2}(1,161)=5,147 ; p=0,076\right]$.

$\mathrm{Na}$ Tabela 4, observamos uma prevalência elevada de pessoas com pontuações sugestivas de déficit cognitivo, considerando o total de pessoas que reside nestas instituições $(67,8 \%)$.

A proporção de pessoas com pontuações sugestivas de déficit cognitivo foi significativamente superior nas respostas sociais de Miranda do Corvo comparado à obtida no Estudo do Perfil do Envelhecimento da População Portuguesa de $2010^{45}$ para ambas as coortes etárias (Tabela 5).

\section{Discussão e Conclusão}

O primeiro objetivo deste estudo foi identificar o perfil cognitivo de uma população a residir na resposta social "Estrutura Residencial para Idosos" no Concelho de Miranda do Corvo. Os dados recolhidos através do MMSE foram analisados por item, por dimensão e para o total da escala. Assim, de entre as seis aptidões avaliadas pelo MMSE, as duas aptidões que apresentaram média mais elevada foram a Retenção e a Linguagem. As médias obtidas em cada um destes itens

Tabela 2. Regressão linear predizendo as pontuações no Mini-Mental State Examination.

\begin{tabular}{|c|c|c|c|c|c|c|}
\hline \multicolumn{7}{|c|}{ Coeficientes $^{\mathrm{a}}$} \\
\hline & \multirow[t]{2}{*}{ Modelo } & \multicolumn{2}{|c|}{ Coeficientes não padronizados } & \multirow{2}{*}{$\begin{array}{c}\begin{array}{c}\text { Coeficientes } \\
\text { padronizados }\end{array} \\
\text { Beta }\end{array}$} & \multirow[t]{2}{*}{$\mathbf{t}$} & \multirow{2}{*}{$\mathbf{p}$} \\
\hline & & B & Erro Padrão & & & \\
\hline \multirow[t]{3}{*}{1} & (Constante) & 25,980 & 5,226 & & 4,971 & 0,000 \\
\hline & Idade & $-0,098$ & 0,060 & $-0,159$ & $-1,626$ & 0,107 \\
\hline & Escolaridade & 1,158 & 0,347 & 0,326 & 3,342 & 0,001 \\
\hline
\end{tabular}

a Variável Dependente: Pontuações brutas no Mini-Mental State Examination.

Tabela 3. Frequências e percentagens de pessoas idosas sem e com pontuações sugestivas de déficit cognitivo (determinadas a partir do Mini-Mental State Examination e do diagnóstico de demência extraído dos prontuários do paciente) segundo a escolaridade $(\mathrm{N}=161)^{\mathrm{a}}$

\begin{tabular}{lccc}
\hline & $\begin{array}{c}\text { Com déficit cognitivo } \\
\mathbf{n}(\%)\end{array}$ & $\begin{array}{c}\text { Sem déficit cognitivo } \\
\mathbf{n}(\%)\end{array}$ & Total \\
\hline Analfabetos & $50(64,1)$ & $28(35,9)$ & $78(100)$ \\
1 a 11 anos de escolaridade & $46(62,2)$ & $28(37,8)$ & $74(100)$ \\
Escolaridade superior a 11 anos & $9(100)$ & $0(0,0)$ & $9(100)$ \\
Total & $105(65,2)$ & $56(34,8)$ & $161(100)$ \\
\hline
\end{tabular}

$\chi^{2}=$ qui-quadrado de Pearson.

ns = não significativo.

${ }^{a} \mathrm{O}$ processo não incluía informação relativa à escolaridade de treze pessoas.

Tabela 4. Frequências e percentagens de pessoas idosas sem e com pontuações sugestivas de déficit cognitivo determinadas a partir do Mini-Mental State Examination (MMSE) e do diagnóstico de demência extraído dos prontuários dos pacientes $(\mathrm{N}=174)$

\begin{tabular}{lccc}
\hline & MMSE & Prontuário do paciente & $\begin{array}{c}\text { MMSE + Prontuário do } \\
\text { paciente }\end{array}$ \\
\cline { 2 - 4 } & $\mathbf{n}(\%)$ & $\mathbf{n}(\%)$ & $\mathbf{N}(\%)$ \\
\hline Déficit cognitivo & $40(41,7)$ & $78(100)$ & $118(67,8)$ \\
Sem déficit cognitivo & $56(58,3)$ & $0(0)$ & $56(32,2)$ \\
Total & $96(100)$ & $78(100)$ & $174(100)$ \\
\hline
\end{tabular}


Tabela 5. Comparação dos nossos resultados com os obtidos pelo estudo do perfil do envelhecimento da população portuguesa -2010 para as duas coortes etárias $(\mathrm{N}=174)$

\begin{tabular}{llccc}
\hline & & $\begin{array}{c}\text { ERPIs de Miranda do Corvo } \\
\text { N (\%) }\end{array}$ & $\begin{array}{c}\text { Perfil Populacional }^{33} \\
\text { N }(\%)\end{array}$ & $\boldsymbol{\chi}^{\mathbf{2}}$ \\
\hline$[65-74]$ & Com déficit cognitivo & $12(60,0)$ & $32(3,6)$ & \\
& Sem déficit cognitivo & $8(40,0)$ & $868(96,4)$ & \multirow{2}{*}{$124,77^{* * *}$} \\
& Total & $20(100)$ & $900(100)$ & \\
\hline$\geq 75$ & Com déficit cognitivo & $103(68,2)$ & $74(12,0)$ & \\
& Sem déficit cognitivo & $48(31,8)$ & $545(88,0)$ & $213,01^{* * *}$ \\
& Total & $151(100)$ & $619(100)$ & \\
\hline Total & Com déficit cognitivo & $115(60,3)$ & $106(7,0)$ & \multirow{2}{*}{$491,22^{* * *}$} \\
& Sem déficit cognitivo & $56(39,7)$ & $1413(93,0)$ & \\
\hline
\end{tabular}

$\chi^{2}=$ qui-quadrado de Fisher ${ }^{* * *} p<0,001$.

do MMSE são de 0,94 $(D P=0,15)$ com $94 \%$ de respostas corretas para a Retenção, e de $0,80(D P$ $=0,14)$ com $80 \%$ de respostas corretas na Linguagem. Quanto à Retenção, o que poderá contribuir para uma possível explicação destes acertos é o fato de, na avaliação desta aptidão, intervirem apenas três palavras e repetidas numa sequência imediata à apresentação do administrador do teste. Neste sentido, Spar e La Rue ${ }^{46}$, quando se pronunciam sobre a má classificação dos doentes de Alzheimer na recordação retardada, afirmam que, para algumas pessoas idosas, a recordação de poucas palavras (uma a três) pode estar dentro dos limites normais. Recordemos que Parente et al. ${ }^{47}$ afirmam que as produções fonológica e sintática se mantêm preservada com a idade. Estudos que evidenciam diferenças referem que existem mudanças cognitivas com o envelhecimento no que concerne a aspetos específicos da linguagem (e.g., complexidade proposicional e sintática na produção da linguagem e conteúdo e ritmo na fluência verbal $)^{48,49}$, mas os aspetos nucleares são robustos ao envelhecimento cerebral (e.g., compreensão do discurso e processamento sintático ${ }^{50,51}$. Ainda no que respeita à Linguagem, o item onde se verificou pior desempenho foi o item "Escrever uma frase com sentido e com sujeito e verbo" com apenas 39\% das respostas corretas. O desempenho pode ser explicado, do nosso ponto de vista, pela escolaridade da coorte geracional inquirida.

Por sua vez, acerca da dimensão Orientação, a pontuação média foi de 0,72 $(D P=0,23)$, contribuindo para esta pontuação o fato de a Orientação Temporal ser um marcador pouco dependente do nível educacional. Um exame minucioso da aptidão cognitiva Orientação Temporal demonstrou diferenças nas pontuações quando comparada com a Orientação Espacial. Neste ponto, é importante notar que a afetação da Orientação Temporal pode estar menos dependente do nível educacional, constituindo-se como um marcador precoce de problemas cognitivos $^{52}$. É também importante lembrar que a componente Orientação Temporal tende a estar afetada na demência de Alzheimer (quando comparada com a demência frontotemporal e com o envelhecimento normal) e que tende a ser mais afetada do que a Orientação Espacial em doentes com demências de Alzheimer e frontotemporal quando comparados com idosos sem demência ${ }^{53}$.

Na quarta posição, apareceu a aptidão cognitiva Atenção e Cálculo, com 46\% dos acertos, enquanto a aptidão Evocação apareceu em penúltima posição ( $5^{\mathrm{a}}$ posição), com $48 \%$ dos acertos. Um fato recorrentemente referido nos trabalhos de investigação nesta aptidão é que os níveis educacionais baixos, como é o caso dos nossos inquiridos, interferem na performance destes itens ${ }^{54,55}$. Note-se ainda que esta tarefa não é a que melhor discrimina entre envelhecimento normal, déficit e demência ${ }^{40}$.

No que diz respeito à Evocação, Ardila et al. ${ }^{56}$ afirmam que pessoas analfabetas necessitam de um maior número de repetições de palavras para as conseguirem memorizar. De igual modo, e segundo Spar e La Rue ${ }^{46}$, os doentes de Alzheimer terão pior pontuação, especialmente na recordação retardada. Em abono dos nossos resultados, Simning et al..$^{57}$ encontraram percentagem seme- 
lhante $(50,3 \%)$ na evocação de três itens no teste Mini-Cog em pessoas idosas institucionalizadas.

De acordo com a análise de Yew et al. ${ }^{53}$, pessoas idosas sem demência revelam sempre alguma perda na tarefa Evocação numa prova semelhante ao MMSE (Addenbrooke's Cognitive Examination). A capacidade visuoconstrutiva apresentou a pontuação mais baixa entre as seis aptidões avaliadas, com uma pontuação média de $0,21(D P=0,41)$, isto é, com apenas $21 \%$ de acertos, concorrendo para esta baixa performance o fato de o ato de escrever/desenhar implicar movimentos motores finos, aptidão adquirida durante o processo de alfabetização. Assim, é compreensível que os analfabetos, cuja taxa é elevada na nossa amostra, enfrentem grandes dificuldades na realização de tarefas com movimentos complexos ${ }^{56}$. Além disso, acresce o fato de que também Simning et al..$^{57}$ encontraram valores altos de incapacidade de realização visuoconstrutiva (teste do desenho do relógio) em pessoas institucionalizadas. Note-se também esta tarefa se inclui entre as que pior discriminam entre envelhecimento normal, déficit e demência ${ }^{40}$.

Entrando no segundo objetivo, os resultados deste estudo, demonstram que o único fator a predizer a pontuação no MMSE foi a escolaridade. Estes resultados estão em linha com os encontrados por Santana et al..$^{58}$ onde foi revelada uma correlação positiva significativa entre o MMSE com a escolaridade $(r=0,24, p=0,01)$. No entanto, se analisarmos a associação entre a variável com "déficit/sem déficit cognitivo" e a escolaridade podemos observar que as pessoas com escolaridade superior a 11 anos apresentam déficit cognitivo. A aparente contradição entre os resultados obtidos na regressão linear e no teste de independência do qui-quadrado serão explicados pela dicotomização das pontuações do MMSE. As pessoas com maior nível de escolaridade apresentam, tendencialmente, pontuações mais elevadas no MMSE quando comparadas com as suas congêneres com habilitações mais baixas, no entanto essas pontuações apesar de mais elevadas são sugestivas de déficit cognitivo para o ponto de corte com escolaridade mais elevada. Adicionalmente, a associação entre o déficit cognitivo e a escolaridade elevada poderá explicar-se pela mediação de outras variáveis, nomeadamente os recursos econômicos, que poderão interferir com a institucionalização, adiando-a. Assim, perspectivamos que nos casos de pessoas idosas com elevado nível educacional, os familiares ativem este tipo de resposta social especialmente quando essas pessoas idosas sofrem de demência ou déficit cognitivo relevante.
No que concerne à idade, apesar desta variável não ter impacto estatisticamente significativo sobre as pontuações no MMSE, constatou-se que a percentagem de déficit cognitivo é maior nas idades mais avançadas. Assim, este é um resultado parcialmente suportado por estudos desta área $^{57,58}$ que encontram associações significativas. No entanto, o não-impacto da idade sobre o estado cognitivo poderá ser explicado, tal como na escolaridade, pela mediação de outras variáveis, nomeadamente os recursos econômicos e comorbilidades ${ }^{5,6}$ que impactam com a institucionalização.

Quanto ao terceiro objetivo, os resultados obtidos através da administração do MMSE permitem verificar que, entre os inquiridos, quarenta pessoas idosas apresentam resultados sugestivos de déficit cognitivo (41,7\%). Se adicionarmos a estes inquiridos os restantes utentes com diagnóstico de demência reportado nos prontuários dos pacientes, este número sobe para cento e dezoito pessoas, ou seja, $67,8 \%$ das pessoas que residem neste tipo de resposta social apresentam pontuações sugestivas de déficit cognitivo. No entanto, a prevalência por nós obtida é muito mais alta do que a do estudo de Simming et al. ${ }^{57}$ que encontraram uma prevalência $27,6 \%$ e $27,1 \%$ para déficit cognitivo. Note-se, no entanto, que no estudo de Simming et al. $.^{57}, 53,2 \%$ dos participantes tinham escolaridade igual ou superior aos 12 anos (não é feita referência a analfabetismo), enquanto, nos nossos inquiridos, somente $5,1 \%$ tinham esse grau de escolaridade, o que vem reforçar a ideia já presente nas análises anteriores da importância da escolaridade na realização de provas neuropsicológicas.

Em resposta ao quarto objetivo, constatou-se que a expressividade das pessoas nesta situação se situa na cifra dos 67,8 pontos percentuais, bem acima do indicado no Estudo do Perfil do Envelhecimento da População Portuguesa de $2010^{45}$. Apesar de a pontuação obtida com o MMSE ter em linha de conta a escolaridade dos respondentes, os resultados do estudo presente podem ser potenciados pela conjugação do analfabetismo com determinadas características personológicas (e.g., alguns estudos salientam o impacto do traço de personalidade neuroticismo no déficit cognitivo $^{59}$ e com condições socioeconômicas experienciadas no curso da vida ${ }^{60}$.

No entanto, a tendência é clara para a presença de déficit cognitivo nas pessoas idosas neste tipo de resposta social. Esta realidade coloca novos desafios societários e organizacionais nos cuidados às pessoas idosas. Com o advento da 
modernidade, a rede de apoio às pessoas idosas com déficit assume práticas cada vez mais institucionalizadas devido às dificuldades de conciliação das famílias - a gestão do tempo e das atividades quotidianas das pessoas parece inconciliável com este tipo de cuidado - e devido às exigências acrescidas que o déficit implica em termos de conhecimentos e ferramentas de estimulação e revitalização cognitivo das pessoas idosas. Os resultados encontrados parecem indiciar, indo ao encontro de outros estudos ${ }^{61}$, que o funcionamento cognitivo das pessoas idosas poderá ser um bom preditor da institucionalização. Outra explicação para esta prevalência é a de que a institucionalização poderá ser um meio facilitador do desenvolvimento do déficit cognitivo. Face à natureza do estudo, transversal não experimental, não poderemos estabelecer causalidade. Não existe evidência se foi a presença de déficit cognitivo prévio que conduziu à institucionalização, tal como Pasquini et al. ${ }^{61}$, Luppa et al. ${ }^{23}$ e Tuokko et al. ${ }^{62}$ mostraram, ou se foi no decurso da institucionalização que surgiu o déficit cognitivo ${ }^{29}$.

Como foi referido na parte introdutória deste artigo, as características organizacionais dos cenários institucionais não estimulam as competências cognitivas dos seus utentes, uma opinião também endossada por Paúl ${ }^{63}$. No entanto estes resultados devem ser refletidos à luz de algumas limitações detectadas. Assim, quando referimos que 78 pessoas idosas tinham diagnósticos de demência no prontuário dos pacientes, esses dados não se referem, na grande maioria dos casos, à forma como os diagnósticos são efetuados, à natureza das condições diagnosticadas (e.g., curso, desenvolvimento, comorbilidade), aos critérios usados nos diagnósticos (e.g., os critérios do Manual de Diagnóstico e Estatística das Doenças Mentais), nem outra informação complementar (e.g., imagiológica ou neuropsicológica).

$\mathrm{Na}$ sequência destes resultados, e apesar das limitações referidas, importa refletir sobre a adequação dos cuidados prestados neste tipo de resposta social, em especial sobre a ausência/escassez de programas de estimulação das funções cognitivas. É, por isso, crucial mudar procedimentos nas atividades quotidianas, concretizando programas que visem a otimização do desempenho cognitivo das pessoas institucionalizadas ${ }^{64}$. Poderá, assim, retardar-se o agravamento de sinais demenciais ou de doenças degenerativas, ao mesmo tempo em que se proporciona uma melhor qualidade de vida às pessoas que residem nestes contextos institucionalizados.

\section{Colaboradores}

F Daniel elaborou o projeto de pesquisa, fez parte da revisão de literatura, desenho da metodologia de análise de dados, estrutura do artigo e escrita da versão final. V Fernandes fez parte da revisão de literatura e recolheu os dados. A Silva fez parte da análise de dados. H Espírito-Santo fez parte da revisão de literatura e escrita da versão final. 


\section{Referências}

1. Ariès P. Une histoire de la vieillesse? Communications. Persée - Portail des revues scientifiques en SHS 1983;37(1):47-54.

2. Instituto Nacional de Estatística (INE). Censos 2011 Resultados Definitivos - Portugal. Lisboa: INE; 2013.

3. PORDATA. População residente: total e por grandes grupos etários: onde há mais e menos jovens, idosos ou pessoas em idade activa? [página na Internet]. 2018 [acessado $2018 \mathrm{Fev}$ 2]. Disponível em: https://www.pordata.pt/Municipios/Popula\%C3\% $\mathrm{A} 7 \% \mathrm{C} 3 \% \mathrm{~A} 3 \mathrm{o}+$ residente+total+e+por+grandes+ grupos+et $\% \mathrm{C} 3 \% \mathrm{~A} 1$ rios- 390

4. Clegg A, Young J, Iliffe S, Rikkert MO, Rockwood K. Frailty in elderly people. Lancet. 2013;381(9868):752762.

5. Júnior FBA. Fragilidade, perfil e cognição de idosos residentes em área de alta vulnerabilidade social. Ciên Saude Colet [periódico na internet]. 2017 Dez [acessado 2018 Fev 2];24(8):3047-3056. Disponível em: http://www.cienciaesaudecoletiva.com. br/artigos/fragilidade-perfil-e-cognicao-de-idosos-residentes-em-area-de-alta-vulnerabilidade-social $/ 16530$ ? id $=16530$

6. Niccoli T, Partridge L. Ageing as a risk factor for disease. Curr Biol Cell Press 2012;22(17):R741-752.

7. Baltes PB, Baltes MM. Psychological perspectives on successful aging: The model of selective optimization with compensation. In: Baltes PB, Baltes MM, editores. Successful aging. Cambridge: Cambridge University Press; 2010. pp. 1-34.

8. Carstensen LL, Fung HH, Charles ST. Socioemotional selectivity theory and the regulation of emotion in the second half of life. Motivation and Emotion 2003;27(2):103-123.

9. Carstensen LL. The influence of a sense of time on human development. Science 2006;312(5782):19131915.

10. Rowe JW, Kahn RL. Successful aging. Gerontologist 1997;37(4):433-440.

11. Cosco TD, Prina AM, Perales J, Stephan BCM, Brayne C. Operational definitions of successful aging: a systematic review. Int Psychogeriatr 2013;26(03):373-381.

12. Castel AD, Balota DA, McCabe DP. Memory efficiency and the strategic control of attention at encoding: Impairments of value-directed remembering in Alzheimer's disease. Neuropsychology 2009;23(3):297-306.

13. Kemper S, Schmalzried R, Herman R, Leedahl S, Mohankumar D. The effects of aging and dual task demands on language production. Neuropsychol Dev Cogn B Aging 2008;16(3):241-259.

14. Scherder EJA, van Paasschen J, Deijen JB, Van Der Knokke S, Orlebeke JFK, Burgers I, Devriese PP, Swaab DF, Sergeant JA. Physical activity and executive functions in the elderly with mild cognitive impairment. Aging Ment Health 2005;9(3):272-280.

15. Alzheimer Europe. Portugal. 2013: The prevalence of dementia in Europe [página na Internet]. 2014 [acessado 2017 Set 18]. Disponível em: https://www. alzheimer-europe.org/Policy-in-Practice2/Country-comparisons/2013-The-prevalence-of-dementia-in-Europe/Portugal
16. Buntinx F, De Lepeleire J, Paquay L, Iliffe S, Schoenmakers B. Diagnosing dementia: No easy job. $B M C$ Fam Pract 2011;12(1):60.

17. Prince MJ, Jackson J, editores. Alzheimer's Disease International: World Alzheimer Report 2009. Alzheimer's Disease International; 2009.

18. Steibel NM, Almeida RMM. Estudo de caso: avaliação neuropsicológica: depressão $\mathrm{x}$ demência. Aletheia 2010;(31):111-120.

19. Daniel F. Profissionalização e qualificação da resposta social "Lar de Idosos" em Portugal. Interaç Soc Nov Modern 2009;9(17):65-74.

20. Daniel F. O último lugar no mundo: Considerações e reconsiderações sobre espaço e velhice. Interaç Soc Nov Modern 2010;1(11):101-112.

21. Bazo MT. Institucionalización de personas ancianas: Un reto sociológico. Reis 1991;53:149-164.

22. Hellstrom Y, Hallberg IR. Perspectives of elderly people receiving home help on health, care and quality of life. Health Soc Care Community 2001;9(2):61-71.

23. Luppa M, Luck T, Weyerer S, König H-H, Brähler E, Riedel-Heller SG. Prediction of institutionalization in the elderly. A systematic review. Age Ageing 2010;39(1):31-38.

24. Luppa M, Sikorski C, Luck T, Ehreke L, Konnopka A, Wiese B, Weyerer S, König HH, Riedel-Heller SG. Age- and gender-specific prevalence of depression in latest-life - Systematic review and meta-analysis. J Affect Disord 2012;136(3):212-221.

25. Paul C, Fonseca AM, Martín I, Amado J. Psychosocial profile of rural and urban elders in Portugal. Eur Psychologist 2003;8(3):160-167.

26. Zank S, Schacke C. Evaluation of geriatric day care units: effects on patients and caregivers. J Gerontol B Psychol Sci Soc Sci 2002;57(4):P348-357.

27. Neri AL, Jorge MD. Atitudes e conhecimentos em relação à velhice em estudantes de graduação em educação e em saúde: subsídios ao planejamento curricular. Estud Psicol (Campinas) 2006;23(2):127-137.

28. Zalik E, Zalar B. Differences in mood between elderly persons living in different residential environments in Slovenia. Psychiatr Danub 2013;25(1):40-48.

29. Silva GF, Espirito-Santo H, Costa M, Cardoso D, Vicente F, Martins S, Lemos L. Reabilitação neuropsicológica grupal de idosos institucionalizados com Défice Cognitivo sem Demência. Rev Port Inv Comp Soc 2015;1(2):15-30.

30. Clare L, Whitaker CJ, Nelis SM, Martyr A, Markova IS, Roth I, Woods RT, Morris RG. Multidimensional assessment of awareness in early-stage dementia: A cluster analytic approach. Dement Geriatr Cogn Disord 2011;31(5):317-327.

31. Teipel S, Heine C, Hein A, Krüger F, Kutschke A, Kernebeck S, Halek M, Bader S, Kirste T. Multidimensional assessment of challenging behaviors in advanced stages of dementia in nursing homes - The insideDEM framework. Alzheimer's \& Dementia: Diagnosis, Assessment \& Disease Monitoring 2017;8:3644. 
32. Canevelli M, Blasimme A, Vanacore N, Bruno G, Cesari M. From Evidence to Action: Promoting a Multidimensional Approach to Mild Cognitive Impairment. J Am Med Dir Assoc 2015;16(8):710-711.

33. Martins N, Caldas PR, Cabral ED, Lins C. Instrumentos de avaliação cognitiva utilizados nos últimos 5 anos em idosos brasileiros. Ciên Saude Colet 2017;24(7):2513-2530.

34. Nitrini RR, Helena Lefèvre B, Mathias SC, Caramelli P, Carrilho PEM, Sauaia N, Massad E, Takiguti C, Silva IO, Porto CS, Magila MC, Scaff M. Testes neuropsicológicos de aplicação simples para o diagnóstico de demência. Arq Neuropsiquiatr 1994;52(4):457-465.

35. Kang S-G, Cho S-J, Ryu S-H, Choi SH, Han S-H, Shim YS, Ko KP, Jeong JH, Lee JY, Lee DW. Normative study of the literacy independent cognitive assessment in illiterate and literate elderly Koreans. Psychiatry Investig 2015;12(3):305-311.

36. Carta Social. Rede de serviços e Equipamentos [página na Internet]. Gabinete de Estratégia e Planeamento do Ministério da Solidariedade, Emprego e Segurança Social do Governo de Portugal; 2014 [acessado 2014 Mar 23]. Disponível em: http://www.cartasocial.pt/ index2.php

37. Folstein MF, Folstein SE, McHugh PR. Mini-mental state. A practical method for grading the cognitive state of patients for the clinician. J Psychiatr Res 1975;12(3):189-198

38. Woodford HJ, George J. Cognitive assessment in the elderly: a review of clinical methods. QJM 2007;100(8):469-484.

39. Melo DM, Barbosa AJG. O uso do Mini-Exame do Estado Mental em pesquisas com idosos no Brasil: uma revisão sistemática. Ciên Saude Colet 2015;20(12):3865-3876

40. Guerreiro M. Testes de rastreio de defeito cognitivo e demência: Uma perspectiva prática. Rev Port Med Geral Famil 2010;26(1):46-53.

41. Guerreiro M, Botelho MA, Leitão O, Garcia C. Adaptação à população portuguesa da tradução do "Mini Mental State Examination" (MMSE). Rev Port Neurol 1994;1:9-10.

42. Moraes C, Pinto JA, Lopes MA, Litvoc J, Bottino CMC. Impact of sociodemographic and health variables on mini-mental state examination in a community-based sample of older people. Eur Arch Psychiatry Clin Neurosci 2010;260(7):535-542.

43. Instituto Nacional de Estatística (INE). Censos 2011 [página na Internet]. [acessado 2018 Fev 2]. Disponível em: https://censos.ine.pt/xportal/xmain?xpid=CENSOS\&xpgid=censos_quadros_populacao

44. Peterson RA. A meta-analysis of Cronbach's coefficient alpha. J Consum Res JSTOR 1994;21(2):381-391.

45. Oliveira CR, Santos-Rosa M, Mota-Pinto A, Botelho MA, Morais A, Veríssimo MT. Estudo do perfil do envelhecimento da população portuguesa. Coimbra: Eurotrials; 2010.

46. Spar JE, La Rue A. Guia prático Climepsi de psiquiatria geriátrica. Lisboa: Climepsi; 2005.

47. Parente M, Saboskinski AP, Ferreira E. Memória e compreensão da linguagem no envelhecimento. Est Interdiscipl Envelhec 1999;1:57-76.
48. Bortfeld H, Leon SD, Bloom JE, Schober MF, Brennan SE. Disfluency rates in conversation: Effects of age, relationship, topic, role, and gender. Lang Speech 2001;44(Pt 2):123-147.

49. Kemper S, Sumner A. The structure of verbal abilities in young and older adults. Psychol Aging 2001;16(2):312-322.

50. Shafto MA, Tyler LK. Language in the aging brain: The network dynamics of cognitive decline and preservation. Science 2014;346(6209):583-587.

51. Tyler LK, Shafto MA, Randall B, Wright P, Marslen-Wilson WD, Stamatakis EA. Preserving syntactic processing across the adult life span: the modulation of the frontotemporal language system in the context of age-related atrophy. Cereb Cortex 2010;20(2):352-364

52. Apolinario D, Lichtenthaler DG, Magaldi RM, Soares AT, Busse AL, Amaral JR, Jacob-Filho W, Brucki SM. Using temporal orientation, category fluency, and word recall for detecting cognitive impairment: the 10-point cognitive screener (10-CS). Int J Geriat Psych 2016;31(1):4-12.

53. Yew B, Alladi S, Shailaja M, Hodges JR, Hornberger $\mathrm{M}$. Lost and forgotten? Orientation versus memory in Alzheimer's disease and frontotemporal dementia. $J$ Alzheimers Dis IOS Press 2013;33(2):473-481.

54. Nielsen TR, Vogel A, Gade A, Waldemar G. Cognitive testing in non-demented Turkish immigrants - comparison of the RUDAS and the MMSE. Scand J Psychol 2012;53(6):455-460.

55. Matallana D, de Santacruz C, Cano C, Reyes P, Samper-Ternent R, Markides KS, Ottenbacher KJ, Reyes-Ortiz CA. The relationship between education level and Mini-Mental State Examination domains among older Mexican Americans. J Geriatr Psychiatry Neurol 2010;24(1):9-18.

56. Ardila AA, Bertolucci PH, Braga LW, Castro-Caldas A, Judd T, Kosmidis MH, Matute E, Nitrini R, Ostrosky-Solis F, Rosselli M. Illiteracy: the neuropsychology of cognition without reading. Arch Clin Neuropsychol 2010;25(8):689-712.

57. Simning A, Conwell Y, van Wijngaarden E. Cognitive impairment in public housing residents living in Western New York. Soc Psychiatry Psychiatr Epidemiol 2013;49(3):477-485.

58. Santana I, Duro D, Lemos R. Mini-Mental State Examination: Avaliação dos novos dados normativos no rastreio e diagnóstico do défice cognitivo [Mini-Mental State Examination: Screening and diagnosis of cognitive decline, using new normative data]. Acta Med Port 2016;29(4):240-248.

59. Boyle LL, Lyness JM, Duberstein PR, Karuza J, King DA, Messing S, Tu X. Trait neuroticism, depression, and cognitive function in older primary care patients. Am J Geriatr Psychiatry 2010;18(4):305-312.

60. Zhang Z, Gu D, Hayward MD. Early life influences on cognitive impairment among oldest old Chinese. J Gerontol B Psychol Sci Soc Sci 2008;63B(1):S25-S33.

61. Pasquini M, Leys D, Rousseaux M, Pasquier F, Henon $H$. Influence of cognitive impairment on the institutionalisation rate 3 years after a stroke. J Neurol Neurosurg Psychiatry 2007;78(1):56-59. 
62. Tuokko H, Frerichs RJ, Graham J, Rockwood K, Kristjansson B, Fisk J, Bergman H, Kozma A, McDowell I. Five-year follow-up of cognitive impairment with no dementia. Arch Neurol 2003;60(4):577-582.

63. Paúl MC. Lá para o fim da vida: idosos, família e meio ambiente. Coimbra: Livraria Almedina; 1997.

64. Tardif S, Simard M. Cognitive stimulation programs in healthy elderly: a review. Int J Alzheimers Dis 2011(3):378934.

Artigo apresentado em 22/10/2017

Aprovado em 24/04/2018

Versão final apresentada em 26/04/2018 